\title{
Independent highly sensitive characterization of asparagine deamidation and aspartic acid
}

isomerization by sheathless CZE-ESI-MS/MS

Rabah Gahoual'1,2, Alain Beck ${ }^{3}$, Yannis-Nicolas François ${ }^{1}$, Emmanuelle Leize-Wagner ${ }^{1}$

${ }^{1}$ Laboratoire de Spectrométrie de Masse des Interactions et des Systèmes (LSMIS), UMR 7140 (UdSCNRS), Université de Strasbourg, Strasbourg, France

2Division of BioAnalytical Chemistry, AIMMS Research Group BioMolecular Analysis, VU University Amsterdam, De Boelelaan 1083, 1081 HV, Amsterdam, The Netherlands

${ }^{3}$ Centre d'immunologie Pierre Fabre, Saint-Julien-en-Genevois, France (www.cipf.com)

Address reprint requests to:

Dr. Rabah Gahoual

Laboratoire de Spectrométrie de Masse des Interactions et des Systèmes

Faculté de chimie

1 rue Blaise Pascal

67008 Strasbourg Cedex

Phone: $+33(0) 368851641$

Mail: rabah.gahoual@etu.unistra.fr 


\begin{abstract}
Amino acids residues are commonly submitted to various physicochemical modifications occurring at physiological $\mathrm{pH}$ and temperature. Post-translational modifications (PTMs) require comprehensive characterization due to their major influence on protein structure and involvement in numerous in vivo process or signaling. Mass spectrometry (MS) has gradually became an analytical tool of choice to characterize PTMs, however some modifications are still challenging due to sample faint modification levels or difficulty to separate an intact peptide from modified counterparts before their transfer to the ionization source. Here we report the implementation of capillary zone electrophoresis coupled to electrospray ionization tandem mass spectrometry (CZE-ESI-MS/MS) by the intermediate of a sheathless interfacing for independent and highly sensitive characterization of asparagine deamidation (deaN) and aspartic acid isomerization (isoD). CZE selectivity regarding deaN and isoD was studied extensively using different sets of synthetic peptides based on actual tryptic peptides. Results demonstrated CZE ability to separate the unmodified peptide from modified homologous exhibiting deaN, isoD or both independently with a resolution systematically superior to 1.29. Developed CZE-ESI-MS/MS method was applied to the characterization of monoclonal antibodies and complex protein mixture. Conserved CZE selectivity could be demonstrated even for complex samples and foremost results obtained showed that CZE selectivity is similar regardless of the composition of the peptide. Separation of modified peptides prior to the MS analysis allowed to characterize and estimate modification levels of the sample independently for deaN and isoD even for peptides affected by both modifications and as a consequence enables to distinguish the formation of Laspartic acid or D-aspartic acid generated from deaN. Separation based on peptide modification allowed, supported by the ESI ionization efficiency provided by CZE-ESI-MS properties, enabled to characterize and estimate studied PTMs with an unprecedented sensitivity and proved the relevance of implementing an electrophoretic driven separation for MS based peptide analysis.
\end{abstract}




\section{Introduction}

Amino acids side chains of proteins and peptides may undergo various chemical reactions occurring at physiological temperature and $\mathrm{pH}$. Those modifications, commonly referred as post-translational modifications (PTMs) includes deamidation, oxidation, phosphorylation, N-terminal acetylation, glutamic acid (Glu) cyclization, isomerization, glycosylation or disulphide bonds exchange for instance [1]. PTMs have a determining influence on biochemical activity of proteins. Indeed, they are involved in inhibition or activation of proteins mainly by modifying their tertiary and quaternary structure likewise acting as biochemical signals transmitter or molecular clocks [2-3]. One of the most frequent PTM, occurring on asparagine (Asn) and marginally on glutamine (Gln) residues, is deamidation which also involves isomerization when forming aspartic acid. Deamidation corresponds to the hydrolysis of the side chain amide bond to a free carboxylic group (Figure 1) [4]. Deamidation kinetics is increased at elevated temperature and $\mathrm{pH}(\geq 8)$ or if the susceptible amino acid is followed by a small, flexible residue such as glycine whose low steric hindrance leaves the amide bond exposed for reaction [5]. In isomerization/racemization, the $\alpha$-carbon of aspartic acid can have two different configurations inducing two configuration isomers: D-aspartic acid (Asp) and L-isoaspartic acid (isoAsp) [4]. Alongside to Asp isomerization resulting from Asn deamidation to both Asp isomers, Asp residues may also spontaneously experience isomerization by the formation of an instable succinimide intermediate. Due to their significant role in biochemical pathways, deamidation and isomerization have been subject to intensive research activities in order to improve their qualitative and quantitative characterization [6-7].

Mass spectrometry (MS) has gradually taken a consequent role in the characterization of PTMs due to its outstanding selectivity and sensitivity. Especially tandem mass spectrometry (MS/MS) allows in various cases to characterize PTMs by attributing the mass difference of the fragment corresponding to the modified amino acid [8]. However MS often needs to be used in combination with a separation technique like nanoflow liquid chromatography (nanoLC) or capillary electrophoresis (CE) in order to limit competition during the electrospray ionization (ESI) process and maximize signal sensitivity. Ion suppression and ionization competition are common phenomena in ESI that may hamper PTMs detection: protein modification is usually faint while major part of the sample remains intact. Without a separation prior to the MS analysis, ionization of a weakly abundant modified peptide would be suppressed during ionization 
leading to the inability to detect the considered PTM. Currently, Asn deamidation and Asp isomerization occurring on protein are characterized by MS using a bottom-up proteomic workflow where the sample of interest is undergoing proteolytic digestion [9]. The peptide mixture generated is then separated and characterized using reverse phase (RP) nanoLC-MS/MS instrumental settings. However, using this analytical strategy, the modification must involve a significant change in hydrophobicity in order to enable the separation of a modified peptide from the intact homologous. Recently, our group developed a method using sheathless capillary zone electrophoresis (CZE) hyphenated to high-resolution MS/MS to perform in a single analysis the simultaneous characterization of different aspects of a protein defining its primary structure [10]. We also demonstrated successfully application of this methodology to perform the biosimilarity assessment of different approved therapeutic mAbs compared to their respective biosimilar candidate [11].

Since a few years, capillary electrophoresis hyphenated to electrospray ionization mass spectrometry (CEESI-MS) is meeting a renewed interest [12-14]. Conventional sheath-liquid CE-ESI-MS interfacing tend to suffer from reduced sensitivity, therefore opposing to one of the major characteristic of MS, while innovative CE-ESI-MS coupling strategies provide an optimal sensitivity especially because of operating flow rates $(<50 \mathrm{~nL} / \mathrm{min})$ enabling the production of a nanoESI [15-16].

In the present work, we demonstrate the interest of using CZE, in complement to the ESI-MS analysis, for the separation of different type of peptide mixtures in order to detect and characterize Asn deamidation as well as Asp isomerization. A comprehensive study was carried out using different sets of synthetic peptides. Synthetic peptides employed are based on actual proteolytic peptides showing the possibility to separate and characterize independently intact peptides from homologous exhibiting Asn deamidation, Asp isomerization even for peptides containing both modifications in the same experiment. CZE selectivity was further studied for the primary structure characterization of the therapeutic mAb trastuzumab (Herceptin®). Trastuzumab is a humanized immunoglobulin gamma 1 (HzlgG-1) directed against the HER2/neu receptor which is overexpressed in about $20-30 \%$ of invasive breast cancer patients [17-18]. A tryptic digest of the mAb was analyzed by sheathless CZE-ESI-MS/MS. MS data were used to characterize several Asn deamidation and Asp isomerization sites of interests. The characteristics provided by the CZE separation are particularly interesting for mAbs characterization because these modifications can influence the potency 
of therapeutic proteins and therefore are categorized as critical quality attributes (CQA) for biotherapeutics [19-20]. Finally a complex peptide mixture was characterize in a similar manner to investigate the conservation of CZE selectivity for complex samples composed of several dozens of proteins. Universal Proteomic Standard 1 (UPS1) was selected as complex peptide mixture to evaluate the analytical platform. The UPS1 is a standard protein mixture designed by the Association of Biomolecular Resources Facilities (ABRF) standard proteomic research group, composed of 48 human proteins ranging from 6 to $83 \mathrm{kDa}$, in equivalent quantities [21]. Sample was spiked with several peptides in order to demonstrate strictly the conserved selectivity of the separation even for complex peptide mixture. Thus we demonstrate the selectivity provided by the electrophoretic separation, between a modified peptide and the intact equivalent likewise emphasizing the exquisite compatibility of CZE conditions to ESI-MS.

\section{Materials \& methods}

\section{Materials}

Chemicals were of analytical grade or high purity grade and purchased from Sigma-Aldrich (Saint-Louis, $\mathrm{MO}$ ) otherwise if not specified. Water used to prepare buffers and sample solutions was obtained using an ELGA purelab UHQ PS water purification system (Bucks, UK). Trastuzumab sample is the EMA approved product and formulation purchased from Roche Diagnostics $\mathrm{GmbH}$ (Penzberg, Germany). Universal Proteomics Standard 1 (UPS1) was purchased from Sigma-Aldrich and RapiGest SF surfactant from Waters (Milford, MA, USA).

\section{Peptide synthesis}

Peptide HT-22 (cf. Table 1) and corresponding modified forms were obtained from Bachem (Bubendorf, Switzerland). These peptides correspond to tryptic fragment 22 of the heavy chain of trastuzumab (position $276-290)$ as well as to possible related degradation products. Purity was analytically controlled in order to be superior to $95 \%$ and masses of each peptide were measured by MALDI-TOF MS. Peptide HT-3 (cf. Table 1) was synthesized at the Centre d'Immunologie Pierre Fabre (Saint-Julien-en-Genevois, France) using Fmoc chemistry. Purity was shown to be superior to $95 \%$ and masses were measured by MALDI- 
TOF MS before use. These peptides correspond to tryptic fragment 3 of the heavy chain of humanized antibody. Sequence and theoretical masses of the peptides and their isoforms are compiled in Table 1.

\section{Trastuzumab sample preparation}

A volume corresponding to $100 \mu \mathrm{g}$ of protein were used form marketed formulation $(21 \mathrm{mg} / \mathrm{mL})$. Samples were first diluted using milliQ water to a final concentration of $6.7 \mu \mathrm{g} / \mu \mathrm{L}$. Samples were then diluted using $0.1 \%$ RapiGest surfactant to reach a final concentration of $3.35 \mu \mathrm{g} / \mu \mathrm{L}$ and heated to $40^{\circ} \mathrm{C}$ for $10 \mathrm{~min}$. Dithiothreitol (DTT) was added to the sample to a final concentration of $25 \mathrm{mM}$. The samples was then heated for a 5 min incubation at $95^{\circ} \mathrm{C}$. After being cooled down to room temperature (RT), iodoacetamide (2-IDA) was added to the sample to a final concentration of $10 \mathrm{mM}$ and the mixture was kept in the dark for $20 \mathrm{~min}$ to allow alkylation of cysteines (Cys) thiol residues. A volume of $1 \mu \mathrm{L}$ of trypsin $(0.5 \mu \mathrm{g} / \mu \mathrm{L})$ was added to the sample which was left at room temperature for $3 \mathrm{~h}$. Finally another $1 \mu \mathrm{L}$ volume of trypsin was added and digestion was performed overnight at $37^{\circ} \mathrm{C}$. After digestion completion, $1 \%(\mathrm{v} / \mathrm{v})$ formic acid (FA) was added to the samples, which were left at RT in order to cleave the surfactant. Samples were finally diluted to a final concentration of $2.2 \mu \mathrm{M}$ of protein using ammonium acetate $50 \mathrm{mM}(\mathrm{pH} 4.0)$.

\section{UPS1 sample preparation}

UPS1 sample was reconstituted first in $0.1 \%$ RapiGest by adding $30 \mu \mathrm{L}$ of the solution and heated to $40^{\circ} \mathrm{C}$ during 10 min. Trypsin was reconstituted by adding a volume of $40 \mu \mathrm{L}$ of milliQ water as advised by manufacturer. DTT was added to the sample to a final concentration of $25 \mathrm{mM}$. Sample was then incubated for $5 \mathrm{~min}$ at a temperature $95^{\circ} \mathrm{C}$. After being cooled down to RT, 2-IDA was added to the sample to a final concentration of $10 \mathrm{mM}$ and sample was kept in the dark for $20 \mathrm{~min}$. A volume of $1 \mu \mathrm{L}$ of trypsin was added to the mixture which was left at RT for $3 \mathrm{~h}$ and another $1 \mu \mathrm{L}$ was added afterward, digestion was carried on overnight at $37^{\circ} \mathrm{C}$. After digestion completion, $1 \% \mathrm{FA}(\mathrm{v} / \mathrm{v})$ was added to the sample and left at RT for 2 hours. A volume of $12 \mu \mathrm{L}$ was sampled and concentrated to an approximate volume of $1 \mu \mathrm{L}$ using vacuum concentration (miVAC, NY, USA).

\section{Capillary zone electrophoresis}


CZE separations were performed using CESI8000 system from Sciex separations (Breas, CA, USA) equipped with a temperature controlled autosampler and a power supply able to deliver up to $30 \mathrm{kV}$. Hyphenation to the mass spectrometer was carried out using the sheathless CE-ESI-MS interfacing equipped on the CESI8000 system. Bare fused-silica (BFS) capillaries (total length $92 \mathrm{~cm} ; 30 \mu \mathrm{m}$ i.d.) with a characteristic porous tip on its final $5 \mathrm{~cm}$ were made available by Sciex separations. New capillaries were flushed at $75 \mathrm{psi}$ (5.17 bar) for 10 min with methanol, $10 \mathrm{~min}$ with $0.1 \mathrm{M}$ sodium hydroxide, then 10 min with $0.1 \mathrm{M}$ hydrochloric acid and water for $20 \mathrm{~min}$. Finally, the capillary was flushed $10 \mathrm{~min}$ at 75 psi with $10 \%$ acetic acid (AA), which was the BGE used for the separation. Unless stated, hydrodynamic injections (410 mbar for $100 \mathrm{sec}$ ) corresponding to a total volume of $110 \mathrm{~nL}$ were used. Separations were performed by applying a voltage of $+20 \mathrm{kV}$.

\section{Sheathless CE-ESI-MS hyphenation}

CESI8000 system was hyphenated to a 5600+ TripleTOF mass spectrometer (AB Sciex, Darmstadt, Germany). The $5600+$ MS is equipped with a hybrid analyzer composed of a succession of quadrupoles followed by a time-of-flight (TOF) mass analyzer. ESI source parameters were set as follows: ESI voltage $-1.75 \mathrm{kV}$, gas supplies (GS1 and GS2) were deactivated, source heating temperature $150^{\circ} \mathrm{C}$ and curtain gas value 2 psi.

\section{Mass spectrometry}

For synthetic peptides analysis, MS/MS experiments were performed in Top10 information dependent acquisition (IDA), accumulation time set to $250 \mathrm{msec}$ for MS scans and $80 \mathrm{msec}$ for MS/MS scan leading to a duty cycle of $1.05 \mathrm{sec}$. Mass/charge ratio (m/z) range was set to $100-2000$ in both MS and MS/MS experiments. For trastuzumab and UPS1 characterization, experiments were carried out in Top20 IDA experiment with similar parameters (duty cycle $1.85 \mathrm{sec}$ ).

MS/MS data analysis 
For protein identification, MS/MS raw data were processed using ProteinPilot software (AB Sciex, Darmstadt, Germany). Database search were carried out using software native algorithm Paragon configured with the following parameters (a) database: Swissprot database, (b) enzyme: Trypsin/P, (c) maximum missed cleavages: 3, (d) variable modifications (amino acid): acetylation (protein $\mathrm{N}$-term), deamidation (Asn), oxidation (Met), carbamidomethylation (Cys), (e) False Discovery Rate (FDR) $<1 \%$. If required, additional data treatment of CESI-MS/MS experiments was performed manually using PeakView software.

\section{Results and Discussions}

\section{Modified peptides separation under CZE}

In order to investigate the selectivity provided under the electrokinetically driven separation, two different set of peptides incorporating the PTMs of interests were synthesized. Characteristics of those peptides are summarized in Table 1. Each peptide set consists of the considered peptide without modification and the similar peptide bearing Asn deamidation, Asp isomerization or both modifications. Note as these peptides are based on actual tryptic peptides C-terminal extremity is composed of either a lysine (Lys) or an arginine (Arg) in order to emphasize the characterization of a tryptic peptides (Table 1). While peptide synthesis allows to obtain independently the same peptide with different Asp conformation. The first set of peptides (HT-22) contains two distinctive modification sites: an Asp residue in position $6\left(D^{6}\right)$ and an Asn residue in position $12\left(\mathrm{~N}^{12}\right)$ which can undergo through isomerization and deamidation/isomerization respectively. Different mixtures of the considered peptides were prepared in order to determine whether CZE enables separation regarding strictly Asn deamidation and Asp isomerization. As emphasized in Figure 2, the separation of a mixture constituted of HT-22 and HT-22 (deaN ${ }^{12}$ ) peptides exhibited two consecutive peaks. Peptide HT-22 exhibits an apparent mobility of $1.91 \cdot 10^{-5} \mathrm{~cm}^{2} \mathrm{~V}^{-1} \mathrm{~s}^{-1}$ while the same peptide with an Asn deamidation exhibited an apparent mobility of $1.93 \cdot 10^{-5} \mathrm{~cm}^{2} \mathrm{~V}^{-1} \mathrm{~s}^{-1}$, leading to a difference of $0.20 \pm 0.015$ min in migration times corresponding to a resolution of 1.31 (Figure 2). To confirm the separation regarding Asn deamidation, an equivalent mixture was prepared using HT-22 (isoD ${ }^{6}$ ) and HT-22 (isoD $6 /$ deaN $^{12}$ ). Similarly, results obtained showed two consecutive peaks with a difference in migration time of $0.20 \pm 0.01$ min. Considering their peak width, CZE separation gives a resolution of 1.38 meaning that CZE enables a 
significant separation of similar peptides which sole difference is an Asn residue experiencing deamidation. Because deamidation leads to a mass shift of 0.984 Da which can be resolved by high resolution MS, both peaks could be unambiguously attributed and collision induced dissociation (CID) MS/MS spectra even allowed to determine the exact amino acid modified (Figure 2 B. C.). Deamidation does not involve a change of the peptide charge in solution at the $\mathrm{BGE} \mathrm{pH} \mathrm{(2.2).} \mathrm{However,} \mathrm{difference} \mathrm{in} \mathrm{migration} \mathrm{times} \mathrm{is} \mathrm{explained}$ by a modification of the hydrodynamic radius of the peptide due to the deamidation. Relevance of the separation of the two peptides is supported by several observations: both peptides are detected with a charge state of $(+2)$ which means that in case of co-migration, the modification would not be characterized due to the overlapping of their respective isotopic profiles. Additionally, samples are commonly showing partial modification which can be extremely weak $(<5 \%)$. The ability to separate the modified peptide from the intact one before ESI ionization is therefore interesting because it prevents competition during the ionization process and increase the sensitivity of the signal.

CZE-ESI-MS experiments were also performed to study the selectivity of the separation regarding Asp isomerization. Separation of a mixture containing peptide HT-22 (deaN ${ }^{12}$ ) and HT-22 (isoD $\mathrm{D}^{6} / \mathrm{deaN}^{12}$ ), as emphasized in Figure 3, showed two consecutive peaks having a migration time difference of $0.40 \pm 0.10$ min and a resolution of 2.24 . The capacity to separate two peptides composed of the same amino acid sequence but with two different conformation of Asp is certainly a huge advantage in that case. MS instrumental settings used in this study do not permit the characterization of Asp isomerization occurring on a peptide from MS or MS/MS data. Indeed isomerization does not involve a difference in the mass of the tryptic peptide meaning that it cannot be directly distinguished from TOF-MS data. Additionally, y/b fragment ions produced in CID are common to both peptides (Figure 3 B. C.). As peptide mass and charge in solution are not impacted by Asp isomerization, separation between HT-22 (deaN ${ }^{12}$ ) and HT-22 (iso $\mathrm{D}^{6} / \mathrm{deaN}^{12}$ ) is attributed to a change in the hydrodynamic radius of the peptide due to the different conformation. Complete separation before ionization enables to unambiguously distinguish peptides isoforms and prevent competition effect during ESI process. Baseline separation obtained suggests CZEESI-MS is particularly appropriate to perform a relative quantification regarding modification levels of the sample. A mixture of peptides HT-22 and HT-22 (isoD6) was also analyzed using the same conditions. Results obtained revealed similarly two consecutive peaks with a migration time difference of $0.45 \pm 0.04$ 
min. Results recorded for both previous separation enable to conclude that CZE selectivity is conditioned by the presence of two different Asp isomers despite the presence of another PTM on the considered peptide.

In order to confirm and further study the selectivity of the separation, similar experiments were performed using a peptide mixture composed peptide HT-3 (Table 1). Peptide HT-3 is constituted of a higher number of amino acids compared to the previously studied peptide. As emphasize in Table 1, peptide HT-3 contains an Asp residue which can experience isomerization. The analysis of a mixture containing the two isoforms of peptide HT-3 showed again two consecutive peaks having a difference in migration times of $0.16 \pm 0.01$ min, those separation characteristics providing a resolution superior to 1.34 . In order to confirm the nature of each peak present on the electropherogram, several mixture of HT-3 peptides isoforms in different ratios were prepared and analyzed. Results demonstrated that unmodified peptide is detected first followed by the modified isoform similarly to peptide HT-22. The electrophoretic separation enables to distinguish the two peptides while their only difference comes from the conformation of a single Asp residue showing that CZE selectivity regarding Asp conformation is not affected by the peptide composition. As mentioned before, peptide HT-22 can be affected simultaneously by Asn deamidation, Asp isomerization or simultaneously both. CZE-ESI-MS/MS analysis of a mixture containing every form of peptide HT-22 was performed. As presented in Figure 4, each forms of the peptide could be separated in CZE with a resolution ranging from 1.29 up to 1.63 therefore showing four independent peaks.

This result is particularly interesting as it was possible to distinguish the four different forms of HT-22 in the same experiment. Separation regarding those minor structural changes of the peptide is explained by the conjugation of two different characteristics provided by CZE separation: the selectivity of the electrokinetically driven separation allowing to distinguish analytes regarding their size, likewise the tremendous efficiency of CZE separation. Indeed peak widths at half height are ranging from 0.12 min down to $0.06 \mathrm{~min}$ for the second peak (Figure 4) representing a number of theoretical plates ranging from 218105 up to 890837 plates.

Selectivity in tryptic digest characterization 
In order to further study CZE selectivity regarding Asn deamidation and Asp isomerization, a tryptic digest of trastuzumab was characterized using similar CZE-ESI-MS conditions. Especially, we demonstrated the ability to systematically characterize, from a single analysis, the complete amino acid sequence of the protein. Alongside glycosylations could be precisely localized and more than 10 glycans structures could be simultaneously identified using MS/MS spectra [10]. The amino acid sequence of peptide HT-22 is actually part of trastuzumab sequence. Isomerization of $\mathrm{HT}-22$ (isoD ${ }^{6}$ ) is described as influencing significantly the immunogenic activity of trastuzumab by modifying higher order structure of the protein and for this reason is considered as a critical quality attribute [22]. As it can be observed on Figure 5, the extracted ion electropherograms (EIEs) corresponding to the different forms of peptide HT-22 showed three consecutive peaks similarly to the previous experiments involving synthetic peptides. The first peak was identified as the intact peptide HT-22 while the second peak exhibiting a mass shift of $0.984 \mathrm{Da}$, was identified as peptide HT-22 (isoD $\mathrm{D}^{6} / \mathrm{deaN}^{12}$ ) confirmed by fragmentation spectra. The third peak was identified as peptide HT-22 (isoD ${ }^{6}$ ). Additionally, migration order is consistent with experiments performed employing synthetic peptides. Results obtained clearly indicate that the selectivity contributing to the separation of peptides with Asn deamidation and/or Asp isomerization is maintained during the analysis of tryptic digests. Respective maximum intensities were used to estimate the relative abundance of each isoform. Results show that a large majority of peptide HT-22 is not modified. MS signal allowed to estimate that $94.7 \%$ of $\mathrm{HT}-22$ is not experiencing any modification while $3.3 \%$ is experiencing Asp isomerization (deaN6/ $\left.\mathrm{D}^{12}\right)$ and $2.0 \%$ both Asp isomerization and Asn deamidation $\left(\mathrm{N} 6 / \mathrm{isoD}^{12}\right)$. Considering the quantity of digested peptides injected and their abundance in MS, HT-22 modified peptides are representing a total amount of $10.6 \mathrm{fmol}$ which reveals the outstanding ionization efficiency provided by the sheathless CZEESI-MS interfacing. The excellent ionization efficiency is attributed by the conjugation of several characteristics. The flow rate generated by the electroosmotic flow is significantly below $50 \mathrm{~nL} / \mathrm{min}$, which is favorable with the formation of a nanoESI [23]. The interesting point emerging from CZE-ESI-MS/MS data is that peptide HT-22 isoform exhibiting deamidation is only HT-22 ( $\left.\mathrm{D}^{6} / \mathrm{deaN}^{12}\right)$. In this case, data obtained allowed to specifically characterize the isoform which is concerned by the modification giving new insights about the conditions of occurrence of this modification. 
Several Asp residues, potentially experiencing isomerization present on the considered mAb were also studied. CZE-ESI-MS/MS data allowed to confirm the separation of the modified peptide from the unmodified counterpart while showing that isomerization levels for trastuzumab is quite limited, systematically inferior to $5 \%$. Trastuzumab contains in total four Asn and six Asp residues which modification are influencing significantly the potency of this therapeutic protein in case of deamidation and/or isomerization. CZE-ESI-MS/MS data demonstrated for each hotspots separation of the intact peptide from modified equivalents allowing to characterize and perform the relative quantification regarding modification levels in a single experiment. Results obtained therefore demonstrate conservation of the selectivity provided by the electrophoretic separation in the case of tryptic digest characterization. Ionization efficiency provided by the instrumental settings and the ability to separate prior to the ionization process peptides having minor modifications, minimizing therefore ion suppression, demonstrated the possibility using sheathless CZE-ESI-MS coupling to detect and characterize those PTMs even in the case of weakly abundant modification levels.

\section{Selectivity in complex sample characterization}

In the last two decades, performance of MS have been tremendously improved in terms of mass accuracy, resolution as well as sensitivity, acquisition rate and dynamic range. Those improvements allow the analysis of more complex samples and the development of shotgun proteomic experiments [24]. A shotgun proteomic experiment was designed using CZE-ESI-MS/MS for the separation of a moderate complex peptide digest. This experiment was designed in order to determine the suitability of CZE as an alternative to nanoLC for complex samples analysis and to investigate the selectivity regarding Asn deamidation and Asp isomerization for this type of experiment. The sample considered in this experiment was UPS1 standard. UPS1 is commonly used to evaluate the performances of analytical platforms especially regarding sensitivity as well as the capacity to identify successfully proteins having significant similarities regarding their amino acid sequences [25-26]. Samples were prepared in order to be compatible with CZE-ESI-MS analysis conditions (cf. Materials \& Methods). During sample preparation, samples were spiked with each forms of peptide HT-22. Injection conditions were adjusted in order to perform CZE-ESI-MS/MS experiments using a quantity of $50 \mathrm{fmol}$ for each protein representing a total quantity of $129 \mathrm{ng}$ of digested 
peptides. Despise the complexity of this peptide mixture, the EIE corresponding to the $\mathrm{m} / \mathrm{z}$ of peptide HT22 and modified homologous showed that the selectivity of the separation is preserved likewise the efficiency of the separation (Figure 6). Indeed resolutions observed during experiments regarding HT-22 isoforms separation were ranging from 1.24 up to 1.92. Likewise in mAbs tryptic digest characterization, the capacity to separate the different forms of peptide HT-22 before their transfer to the ionization source can prevent competition effect, therefore maintaining an optimal sensitivity and preventing the introduction of biases during relative quantification. CZE-ESI-MS/MS data were additionally used to identify proteins composing the mixture and assess the performances of the sheathless CZE-ESI-MS coupling. Results demonstrated that a single analysis of UPS1 sample led to the identification of all 48 proteins composing the sample through database search algorithm. Strictly relying on database search algorithm, maximum sequence coverage was $73 \%$ for lactotransferin $\left(M_{t h}=78289 \mathrm{Da}\right)$ while mean sequence coverage was 35 \%. Results obtained show the exceptional adequacy of CZE separation in concomitance with high resolution MS for the characterization of complex samples in shotgun bottom-up proteomic experiments as it was also showed by different groups [12-27]. This characteristic explains the excellent results regarding sequence coverage obtained which allowed to distinguish and identify independently proteins having significant parts of their amino acid sequences in common. Such properties of the sheathless CZE-ESIMS/MS analysis, in particular the selectivity provided by CZE separation enables the characterization of lowly abundant PTMs even for a complex sample of digested proteins, giving the opportunity in some cases to dispense with time and sample consuming enrichment steps during sample preparation [28]. In this work, CZE selectivity allowed to distinguish especially several peptides having different isomers of aspartic acid, enriching the level of the characterization which could be achieved in a conventional shotgun bottom-up proteomic experiment considering the type of mass analyzer used during the experiments.

\section{Conclusions}

In this work, we describe the use of sheathless CZE-ESI-MS/MS to improve the characterization of Asn deamidation and Asp isomerization of peptides in bottom-up proteomic experiments. CZE separation selectivity regarding those modifications was extensively investigated using several sets of synthetic model 
peptides. Results demonstrated that CZE conditions employed allowed to separate an intact peptide from the different homologous experiencing Asn deamidation, Asp isomerization or both modifications independently with a resolution systematically higher than 1.29. Selectivity is attributed to the electrokinetically driven separation mechanism of CZE which enables separation of analytes exhibiting different hydrodynamic radii. Separation of peptide isoforms experiencing Asn deamidation and Asp isomerization provides an optimal sensitivity and prevent the introduction of biases that may hamper estimation of sample modification levels. This characteristics was emphasized in the experiments by the ability to detect weak modification levels inferior to $5 \%$ and the possibility to estimate independently modification levels for several PTMs occurring on the same peptide. Results proved the conservation of CZE selectivity even in the case of characterization of complex samples. Additionally, sheathless CZE-ESIMS/MS data allowed to successfully identify all proteins composing the UPS1 sample in a single analysis using a total injection of $129 \mathrm{ng}$ of digested proteins with excellent sequence coverage up to $73 \%$ allowing to identify respectively proteins having extended amino acid sequence homology.

Results obtained demonstrate the adequacy of CZE-ESI-MS/MS conditions with bottom-up proteomics experiments while providing an outstanding sensitivity for detection and identification of faintly abundant PTMs by favoring ionization efficiency. Implementation of CZE separation prior to the ESI-MS analysis allowed to enrich the level of characterization in certain cases. Electrophoretic separation of modified peptides from their intact counter parts, even for modifications inducing only a conformation of a single amino acid appears as a valuable asset for biological samples characterization. Therefore CZE-ESI-MS/MS analysis can be envisage alongside to conventional shotgun proteomics experiments in order to enrich the level of characterization which can be achieved for a given complex sample by taking advantage of the respective separation selectivity provided by reverse phase liquid chromatography and capillary zone electrophoresis.

\section{Acknowledgments}

Authors would like to thank Sciex separations Inc. for lending a CESI8000 system, Dr. M. Anselme and Dr. S. Locke for their support. LSMIS would like to thank P. Hammann, L. Kuhn and J. Chicher (Institut de Biologie Moléculaire et Cellulaire, Strasbourg, France) for stimulating discussions regarding bottom-up 
proteomics experiments. The authors would like also to express their gratitude to Dr. E. Wagner-Rousset, MC. Janin-Bussat, O. Colas and Dr. Christine Klinguer-Hamour (Centre d'immunologie Pierre Fabre, St Julien en Genevois, France) for helpful discussions around peptide and antibody structural characterization.

\section{References}

1. Walsh, C. T.; Garneau-Tsodikova, S.; Gatto, G. J., Protein Posttranslational Modifications: The Chemistry of Proteome Diversifications. Angewandte Chemie International Edition, 2005, 44.

2. Corti, A.; Curnis, F., Isoaspartate-dependent molecular switches for integrin-ligand recognition. Journal of cell science, 2011, 124.

3. Robinson, N. E.; Robinson, A. B., Molecular clocks. Proceedings of the National Academy of Sciences, 2001, 98.

4. Geiger, T.; Clarke, S., Deamidation, isomerization, and racemization at asparaginyl and aspartyl residues in peptides. Succinimide-linked reactions that contribute to protein degradation. Journal of Biological Chemistry, 1987, 262.

5. Chelius, D.; Rehder, D. S.; Bondarenko, P. V., Identification and Characterization of Deamidation Sites in the Conserved Regions of Human Immunoglobulin Gamma Antibodies. Analytical Chemistry, 2005, 77.

6. Wold, F., In Vivo Chemical Modification of Proteins (Post-Translational Modification). Annual Review of Biochemistry, 1981, 50.

7. Shang, J.; Yu, J.; Qin, Y.; Jin, H.; Cai, H.; Guo, X., Fragmentations of the heptapeptides containing $\alpha$ - or $\beta$-aspartate by ESI-MS and low-energy CAD. Journal of Mass Spectrometry, 2010, 45.

8. Yates, J. R.; Eng, J. K.; McCormack, A. L.; Schieltz, D., Method to Correlate Tandem Mass Spectra of Modified Peptides to Amino Acid Sequences in the Protein Database. Analytical Chemistry, 1995, 67.

9. Fornelli, L.; Ayoub, D.; Aizikov, K.; Beck, A.; Tsybin, Y. O., Middle-Down Analysis of Monoclonal Antibodies with Electron Transfer Dissociation Orbitrap Fourier Transform Mass Spectrometry. Analytical Chemistry, 2014, 86.

10. Gahoual, R.; Busnel, J.-M.; Beck, A.; François, Y.-N.; Leize-Wagner, E., Full Antibody Primary Structure and Microvariant Characterization in a Single Injection Using Transient Isotachophoresis and Sheathless Capillary Electrophoresis-Tandem Mass Spectrometry. Analytical Chemistry, 2014, 86.

11. Gahoual, R.; Biacchi, M.; Chicher, J.; Kuhn, L.; Hammann, P.; Beck, A.; Leize-Wagner, E.; François, Y. N., Monoclonal antibodies biosimilarity assessment using transient isotachophoresis capillary zone electrophoresis-tandem mass spectrometry. mAbs, 2014, 6.

12. Sun, L.; Hebert, A. S.; Yan, X.; Zhao, Y.; Westphall, M. S.; Rush, M. J. P.; Zhu, G.; Champion, M. M.; Coon, J. J.; Dovichi, N. J., Over 10000 Peptide Identifications from the HeLa Proteome by Using SingleShot Capillary Zone Electrophoresis Combined with Tandem Mass Spectrometry. Angewandte Chemie International Edition, 2014, 53.

13. Han, X.; Wang, Y.; Aslanian, A.; Bern, M.; Lavallée-Adam, M.; Yates, J. R., Sheathless Capillary Electrophoresis-Tandem Mass Spectrometry for Top-Down Characterization of Pyrococcus furiosus Proteins on a Proteome Scale. Analytical Chemistry, 2014, 86.

14. Li, Y.; Compton, P. D.; Tran, J. C.; Ntai, I.; Kelleher, N. L., Optimizing capillary electrophoresis for top-down proteomics of 30-80 kDa proteins. PROTEOMICS, 2014, 14.

15. Bonvin, G.; Veuthey, J. L.; Rudaz, S.; Schappler, J., Evaluation of a sheathless nanospray interface based on a porous tip sprayer for CE-ESI-MS coupling. Electrophoresis, 2012, 33. 
16. Sun, L.; Zhu, G.; Zhao, Y.; Yan, X.; Mou, S.; Dovichi, N. J., Ultrasensitive and Fast Bottom-up Analysis of Femtogram Amounts of Complex Proteome Digests. Angewandte Chemie, 2013, 125.

17. Hudis, C. A., Trastuzumab - Mechanism of Action and Use in Clinical Practice. New England Journal of Medicine, 2007, 357.

18. Carter, P.; Presta, L.; Gorman, C. M.; Ridgway, J. B.; Henner, D.; Wong, W. L.; Rowland, A. M.; Kotts, C.; Carver, M. E.; Shepard, H. M., Humanization of an anti-p185HER2 antibody for human cancer therapy. Proceedings of the National Academy of Sciences, 1992, 89.

19. Eon-Duval, A.; Broly, H.; Gleixner, R., Quality attributes of recombinant therapeutic proteins: An assessment of impact on safety and efficacy as part of a quality by design development approach. Biotechnology Progress, 2012, 28.

20. Harris, R. J.; Kabakoff, B.; Macchi, F. D.; Shen, F. J.; Kwong, M.; Andya, J. D.; Shire, S. J.; Bjork, N.; Totpal, K.; Chen, A. B., Identification of multiple sources of charge heterogeneity in a recombinant antibody. Journal of Chromatography B, 2001, 752.

21. Jian, L.; Niu, X.; Xia, Z.; Samir, P.; Sumanasekera, C.; Mu, Z.; Jennings, J. L.; Hoek, K. L.; Allos, T.; Howard, L. M.; Edwards, K. M.; Weil, P. A.; Link, A. J., A Novel Algorithm for Validating Peptide Identification from a Shotgun Proteomics Search Engine. Journal of Proteome Research, 2013, 12.

22. Walsh, G., Post-translational modifications of protein biopharmaceuticals. Drug Discovery Today, 2010, 15.

23. Busnel, J.-M.; Schoenmaker, B.; Ramautar, R.; Carrasco-Pancorbo, A.; Ratnayake, C.; Feitelson, J. S.; Chapman, J. D.; Deelder, A. M.; Mayboroda, O. A., High Capacity Capillary Electrophoresis-Electrospray Ionization Mass Spectrometry: Coupling a Porous Sheathless Interface with Transient-Isotachophoresis. Analytical Chemistry, 2010, 82.

24. Bensimon, A.; Heck, A. J. R.; Aebersold, R., Mass Spectrometry-Based Proteomics and Network Biology. Annual Review of Biochemistry, 2012, 81.

25. Tabb, D. L.; Vega-Montoto, L.; Rudnick, P. A.; Variyath, A. M.; Ham, A.-J. L.; Bunk, D. M.; Kilpatrick, L. E.; Billheimer, D. D.; Blackman, R. K.; Cardasis, H. L.; Carr, S. A.; Clauser, K. R.; Jaffe, J. D.; Kowalski, K. A.; Neubert, T. A.; Regnier, F. E.; Schilling, B.; Tegeler, T. J.; Wang, M.; Wang, P.; Whiteaker, J. R.; Zimmerman, L. J.; Fisher, S. J.; Gibson, B. W.; Kinsinger, C. R.; Mesri, M.; Rodriguez, H.; Stein, S. E.; Tempst, P.; Paulovich, A. G.; Liebler, D. C.; Spiegelman, C., Repeatability and Reproducibility in Proteomic Identifications by Liquid Chromatography-Tandem Mass Spectrometry. Journal of Proteome Research, 2010, 9.

26. Ivanov, A. R.; Colangelo, C. M.; Dufresne, C. P.; Friedman, D. B.; Lilley, K. S.; Mechtler, K.; Phinney, B. S.; Rose, K. L.; Rudnick, P. A.; Searle, B. C.; Shaffer, S. A.; Weintraub, S. T., Interlaboratory studies and initiatives developing standards for proteomics. PROTEOMICS, 2013, 13.

27. Wang, Y.; Fonslow, B. R.; Wong, C. C. L.; Nakorchevsky, A.; Yates, J. R., Improving the Comprehensiveness and Sensitivity of Sheathless Capillary Electrophoresis-Tandem Mass Spectrometry for Proteomic Analysis. Analytical Chemistry, 2012, 84.

28. Sarg, B.; Faserl, K.; Kremser, L.; Halfinger, B.; Sebastiano, R.; Lindner, H. H., Comparing and Combining Capillary Electrophoresis Electrospray Ionization Mass Spectrometry and Nano-Liquid Chromatography Electrospray Ionization Mass Spectrometry for the Characterization of Posttranslationally Modified Histones. Molecular \& Cellular Proteomics, 2013, 12. 


\section{Figures}

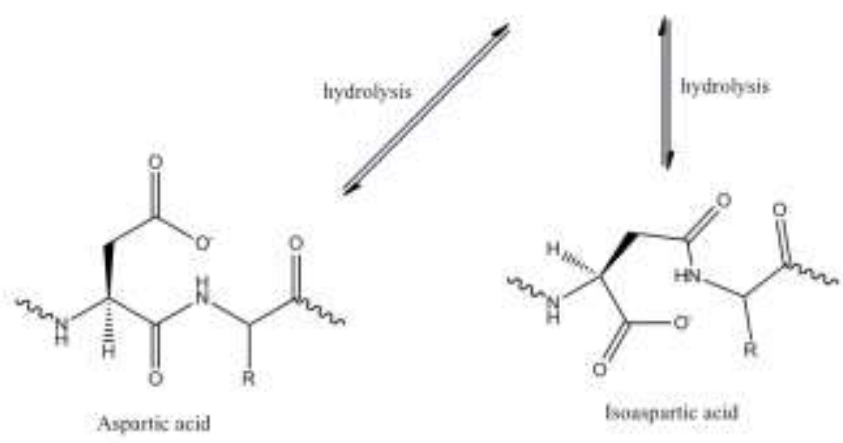

Figure 1 - Representation of nonenzymatic deamidation leading to the formation of aspartic acid and isoaspartic acid. 


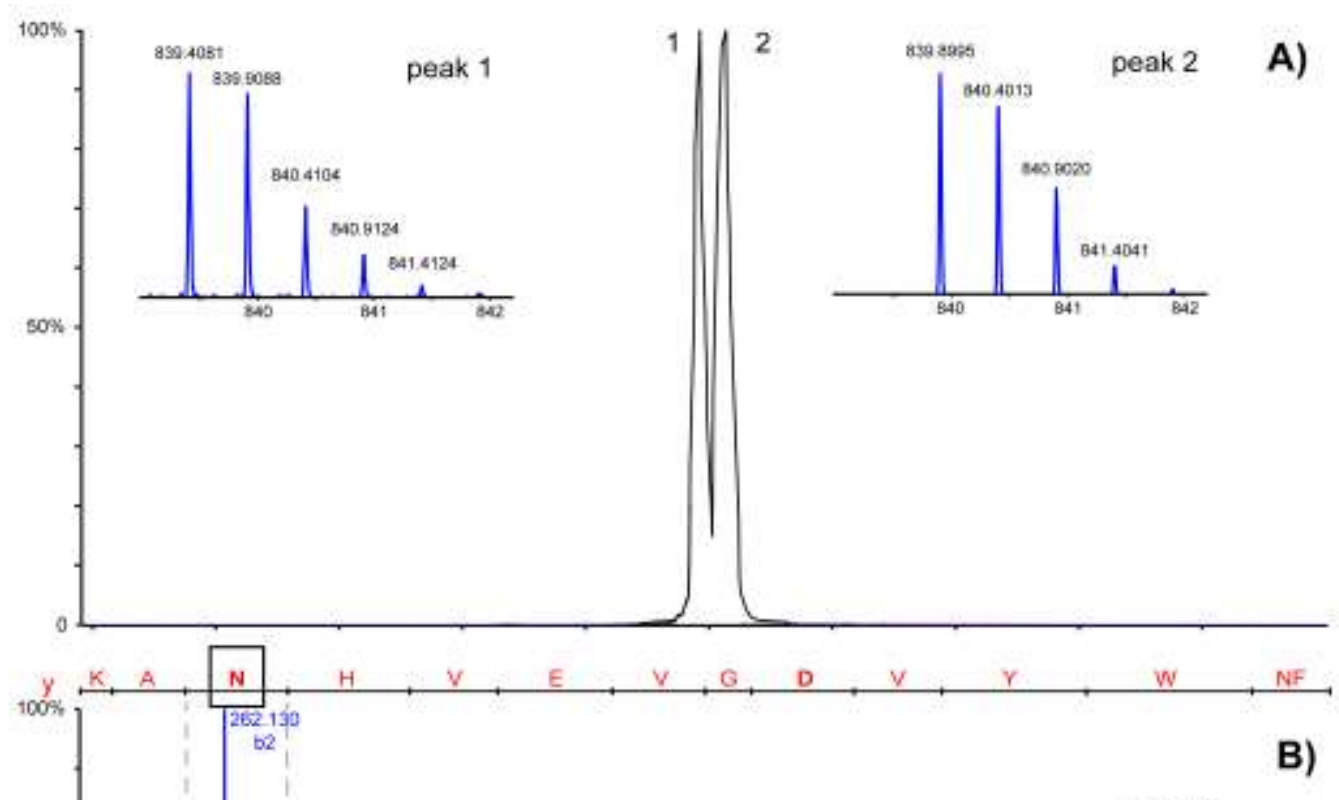

peak 1

B)

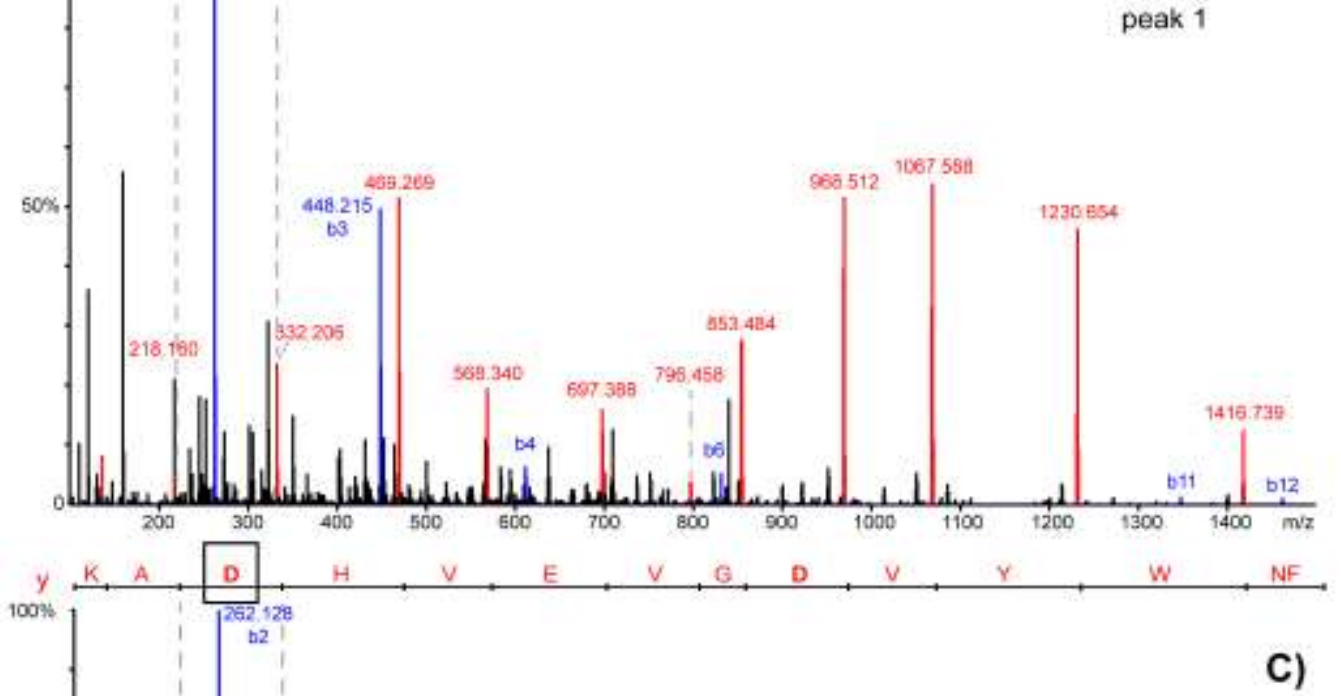

peak. 2

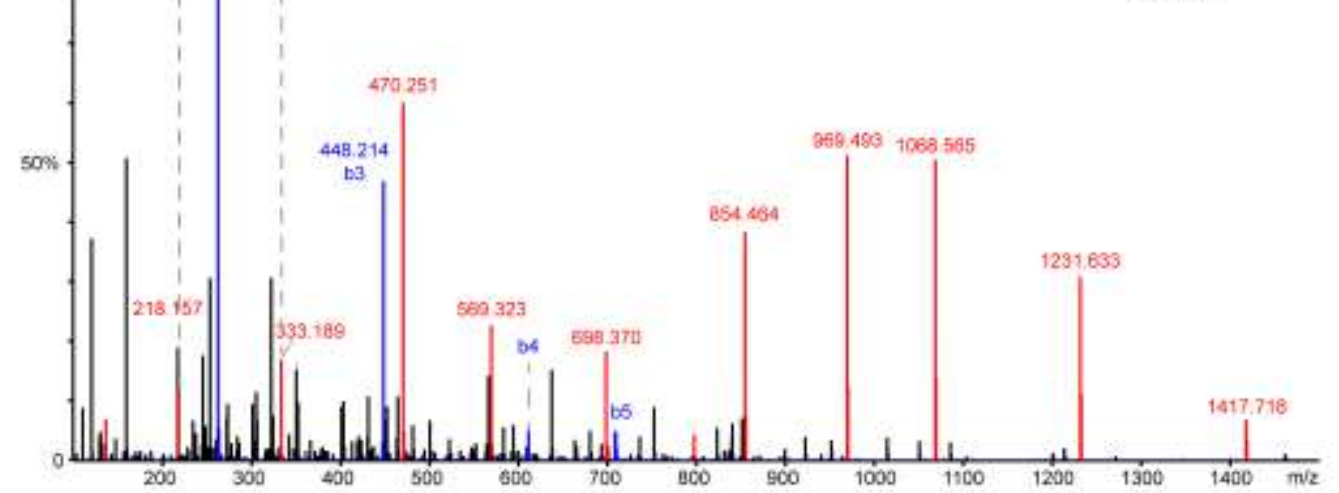

Figure 2 - A) Extracted ion electropherograms (EIEs) for the $\mathrm{m} / \mathrm{z}$ values corresponding to peptide HT-22 and peptide HT-22 (deaN ${ }^{12}$ ) with MS isotopic profiles corresponding to each peak. B) MS/MS spectrum corresponding to peptide HT-22. C) MS/MS spectrum corresponding to peptide HT-22 (deaN ${ }^{12}$ ). 


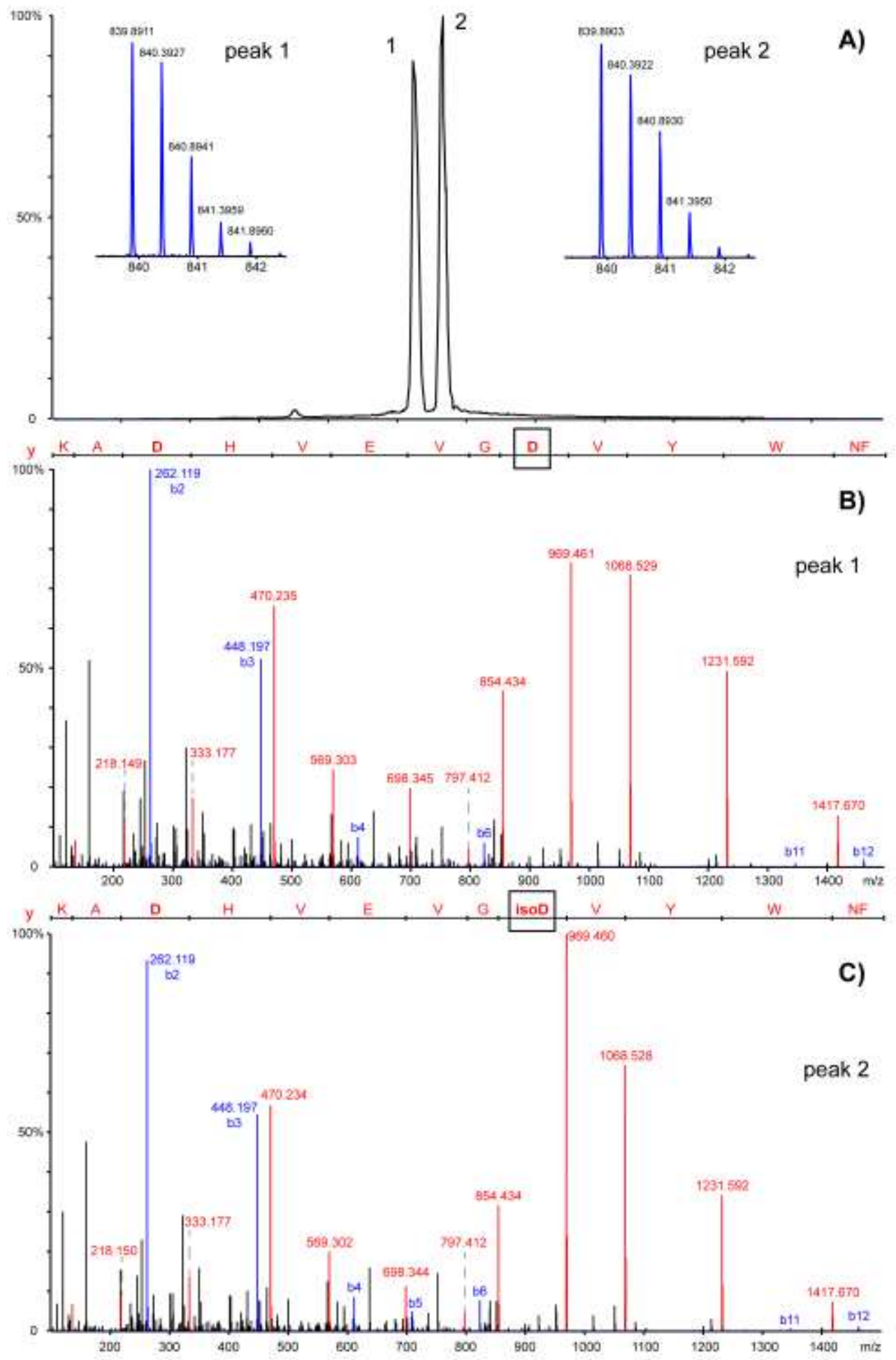

Figure 3 - A) EIE of $\mathrm{m} / \mathrm{z}$ value corresponding to peptide HT-22 ( $\left.\mathrm{D}^{6} / \mathrm{deaN}^{12}\right)$ and peptide HT-22 (iso $\mathrm{D}^{6} / \mathrm{deaN}^{12}$ ) with $\mathrm{MS}$ isotopic profiles corresponding to each peak. B) MS/MS spectrum corresponding to peptide $\mathrm{HT}^{\mathrm{T}}-22$ ( $\mathrm{D}^{6} / \mathrm{deaN}^{12}$ ). C) MS/MS spectrum corresponding to peptide $\mathrm{HT}^{-22}$ (isoD $\mathrm{D}^{6} / \mathrm{deaN}^{12}$ ). 


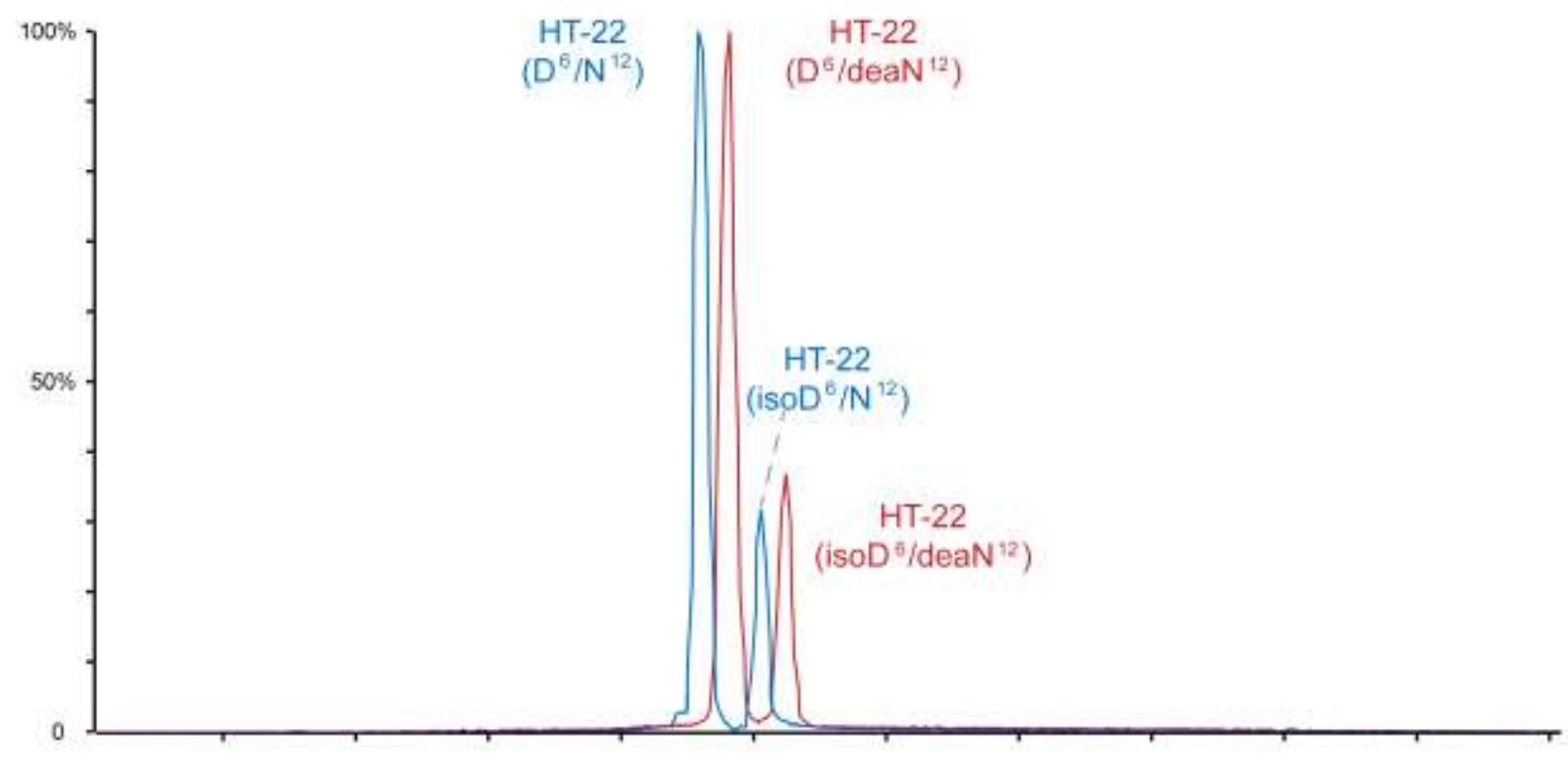

Figure 4 - EIEs of $\mathrm{m} / \mathrm{z}$ values corresponding HT-22 synthetic peptide mixture showing: peaks $1 \mathrm{HT}-22$, peak $2 \mathrm{HT}-22\left(\mathrm{D}^{6} / \mathrm{deaN}^{12}\right)$, peak $3 \mathrm{HT}-22$ (isoD $\mathrm{D}^{6} \mathrm{~N}^{12}$ ) and peak $4 \mathrm{HT}-22$ (isoD $\left./ \mathrm{deaN}^{12}\right)$.

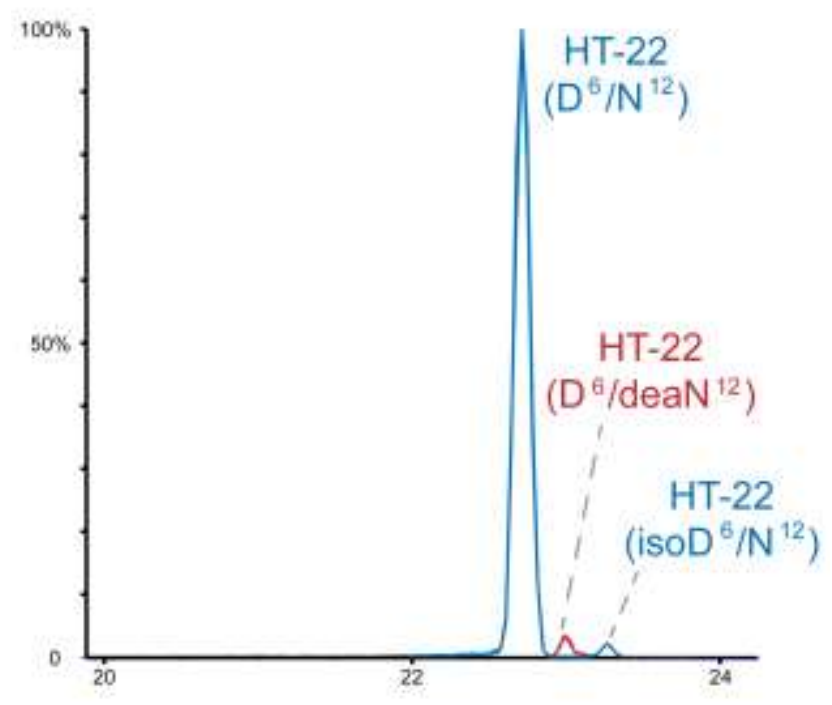

Figure 5 - ElEs of $\mathrm{m} / \mathrm{z}$ values corresponding to the different forms of peptide HT-22 in trastuzumab characterization experiment by CZE-ESI-MS/MS. 




Figure 6 - ElEs of $\mathrm{m} / \mathrm{z}$ values corresponding to different forms of synthetic peptides HT-22 spiked in Universal Proteomic Standard 1 (UPS1) peptide mixture analyzed by sheathless CZE-ESI-MS/MS.

Tables

\begin{tabular}{|c|c|c|c|c|}
\hline Denomination & sequence & modification(s) & denomination & $M_{r}(\mathrm{Da})$ \\
\hline \multirow[t]{4}{*}{ HT-22 } & FNWYVDGVEVHNAK & none & HT-22 & 1676.7947 \\
\hline & FNWYVDGVEVHDAK & deaN & HT-22 (deaN $\left.{ }^{12}\right)$ & 1677.7788 \\
\hline & FNWYVisoDGVEVHNAK & isoD & HT-22 (isoD ${ }^{6}$ ) & 1676.7947 \\
\hline & FNWYVisoDGVEVHDAK & isoD / deaN & HT-22 (iso ${ }^{6} /$ deaN $^{12}$ ) & 1677.7788 \\
\hline \multirow[t]{2}{*}{ HT-3 } & GLEWIGYISYDGGNNYKPSLK & none & HT-3 & 2417.1904 \\
\hline & GLEWIGYISYisoDGTNNYKPSLK & isoD & HT-3 (isoD) & 2417.1904 \\
\hline
\end{tabular}

Table 1-Compilation of different forms of synthetic peptides used to study CZE-ESI-MS/MS method selectivity regarding Asn deamidation and Asp isomerization (cf. Materials and methods). 